\title{
Research potential of food and nutrition in the Family Health Strategy: A structured review
}

\author{
Potencialidades da pesquisa em alimentação \\ e nutrição na Estratégia de Saúde da
}

Família: uma revisão estruturada

Ivana Aragão Lira VASCONCELOS 1

Leonor Maria Pacheco SANTOS²

\section{A B S T R A C T}

\section{Objective}

Determine the profile of research groups and publications with food and nutrition-related actions promoted by the Family Health Strategy in Brazil since 1994.

\section{Methods}

Two procedures were used: structured review and research group search. The former searched the databases Web of Science, Medline, Lilacs, SciELO and Embase, and followed the principles that guide systematic reviews in the Cochrane Collaboration. The references of the selected articles were also consulted. The research groups were searched in the Research Group Directory of the National Council for Scientific and Technological Development.

\section{Results}

A total of 54 articles published between 2002 and 2012 in 20 different journals were identified. Ten of these were retrieved from the references section of other articles. Focusing mostly on children from the Southeast region, these studies were coordinated by dieticians, nurses, and physicians. Diabetes Mellitus, high blood pressure, and breastfeeding were the most common topics $(n=23)$. The quantitative methodology was employed by 42 articles, most about diagnoses. Only five research groups studied the Family Health Strategy, despite the growing number of studies in the area over the years.

\footnotetext{
${ }^{1}$ Universidade de Brasília, Departamento de Nutrição, Programa de Pós-Graduação em Nutrição Humana. Campus Universitário Darcy Ribeiro, Asa Norte, 70910-900, Brasília, DF, Brasil. Correspondência para/Correspondence to: IAL VASCONCELOS. E-mail:<ivana@unb.br>.

${ }^{2}$ Universidade de Brasília, Faculdade de Ciências da Saúde, Departamento de Saúde Coletiva. Brasília, DF, Brasil.
} 


\section{Conclusion}

Despite the growing scientific production, the findings of this structured review indicate that few studies focused on food and nutrition in the Family Health Strategy, probably because of the existence of few research groups in the country. More comprehensive and consistent studies on the topic are needed.

Indexing terms: Family health. Feeding. Nutrition public health. Primary health care. Research groups. Review literature as topic.

\section{RE S U M O}

\section{Objetivo}

Traçar o perfil dos grupos de pesquisa e das publicações que apresentem ações relacionadas à alimentação e nutrição no âmbito da Estratégia Saúde da Família no Brasil a partir do ano de 1994.

\section{Métodos}

Dois procedimentos foram explorados: a revisão estruturada e a busca de grupos de pesquisa. A primeira explorou as bases Web of Science, Medline, Lilacs, SciELO e Embase, seguiu os princípios que orientam as revisões sistemáticas da Colaboração Cochrane. Foram ainda consultadas as referências dos artigos selecionados. A busca de grupos de pesquisa foi feita por meio do Diretório de Grupos de Pesquisa do Conselho Nacional de Desenvolvimento Científico e Tecnológico.

\section{Resultados}

Foram encontrados 54 artigos originais, sendo 10 identificados na lista de referências, publicados entre os anos de 2002 a 2012 em 20 diferentes periódicos. Sua maior parte foi realizada na Região Sudeste, sob coordenação de nutricionistas, enfermeiros e médicos, com crianças como sujeitos de pesquisa. Diabetes Mellitus, Hipertensão e Aleitamento materno foram os temas mais encontrados ( $n=23$ ). Foram identificados 42 artigos de pesquisa quantitativa, em sua maioria sobre diagnóstico. Foram encontrados apenas cinco grupos de pesquisa com o tema na Estratégia Saúde da Família, apesar do aumento de pesquisas na área ao longo dos anos.

\section{Conclusão}

Apesar da produção crescente, os achados desta revisão estruturada indicaram poucos estudos com foco em alimentação e nutrição no contexto da Saúde da Família, provavelmente devido à existência de poucos grupos de pesquisa no País. É necessário fomentar estudos sobre o tema de maneira mais ampla e consistente.

Termos de indexação: Saúde da família. Alimentação. Nutrição em saúde pública. Atenção primária à saúde. Grupos de pesquisa. Literatura de revisão como assunto.

\section{INTRODUCTION}

In the last four decades, the Brazilian population changed its nutritional profile, decreasing the prevalence of childhood malnutrition (although it still persists in lowincome families from the Brazilian North Region), the adolescent and adult underweight prevalences, and the stunting prevalence; and increasing the overweight and obesity prevalences in the country, especially in the last decade ${ }^{1}$. The coexistence of these problems with the persistence of high prevalences of nutritional deficiencies in children under five years of age and women of childbearing age, such as hypovitaminosis $\mathrm{A}(17.4 \%$ and $12.3 \%)$ and anemia (20.9\% and $29.4 \%)^{2}$ characterizes the nutritional transition and the double disease burden ${ }^{3}$.

This situation can be explained by the social and economic transformations that influenced lifestyles, changing the food environment and reducing physical activity. The Pesquisa de Orçamentos Familiares (POF, Family Budget Survey) of 2008-20094 found the addition of lownutrient high-energy foods into the traditional Brazilian diet: more than $90 \%$ of the population had low produce (fruits and non-starchy vegetables) intake and $60 \%$ to $80 \%$ of the population had excessive sugar, sodium, and saturated fat intakes ${ }^{4}$. 
On the other hand, the implementation of policies, and encouragement and support programs promoted breastfeeding, increasing the number of breastfed children and the duration of exclusive and total breastfeeding ${ }^{2}$.

In parallel, the prevalences of moderate and severe food insecurity decreased between the 2004 Pesquisa Nacional por Amostras em Domicilios (PNAD, National Household Sample Survey) and the 2009 PNAD, being 6.5\% and $5.0 \%$, respectively, in the latter ${ }^{5}$. This decrease was confirmed by the 2008-2009 POF, which found a subtle increase in household satisfaction with the amount and quality of the foods $(64.4 \%$ and $35.0 \%$ ) in relation to the 2002-2003 POF $(53.0 \% \text { and } 26.5 \%)^{6}$.

Along with poor food choices, the 2008 PNAD found that only $28.2 \%$ of the population aged more than 14 years was physically active ${ }^{6}$. The Vigilância de Fatores de Risco e Proteção para Doenças Crônicas por Inquérito Telefônico (Vigitel) 2011 found a leisure-time physical activity rate in adults of $30.0 \%$ and a transportation-related physical activity rate of $17.0 \%$.

Given the complexity of the Brazilian nutritional scenario, which tends to increase the incidence and prevalence of chronic NonCommunicable Diseases $(\mathrm{NCD})^{8,9}$, health care personnel need to be trained to ensure the comprehensiveness of care ${ }^{10-12}$. Food and nutrition actions are also capable of favoring the inclusion of health promotion in the political agenda and contribute to this goal10-12.

In this line, the Brazilian State officially advanced by passing the National Health Promotion Policy ${ }^{13}$ in 2006 to promote quality of life and reduce health vulnerability and risk; the National Primary Health Care Policy also in 2006 ${ }^{14}$, which prioritized the Family Health Strategy as the definitive proposal for redirecting the health system; and the National Food and Nutrition Policy, in 1999, revised in $2010^{15}$, to improve the population's diet, nutrition, and health by promoting healthy food practices, food and nutrition surveillance, and preventing and comprehensive care.

Given the various determinants and the combination or overlap of food and nutritionrelated problems, preventive actions should attempt an integral and efficiency care instead of the conventional and fragmented practices ${ }^{11,16}$. Furthermore, the institutional nutrition network in the Sistema Único de Saúde (SUS, Unified Health Care System) became stronger, turning primary care into a potential space for the development of actions that encourage and support healthy eating habits and physical activity $^{12}$.

In the context of comprehensive health care, the Family Health Strategy emerges in 1994 as an innovative proposal to reorganize the health-care model, a proposal capable of establishing a set of political, social, and economic health-promoting ideas that require: uninterrupted intersectoral partnership; interdisciplinary coordination of actions to treat and prevent disease, and promote health; higher education encouraging multidisciplinary care; and health education emphasized as a reflection-action strategy for promoting citizenship and solidarity ${ }^{17}$.

These challenges indicate the importance of proposing the Family Health Strategy as a new model of care and a potential and coherent space for the inclusion and establishment of food and nutrition actions with a cross-sectional approach ${ }^{10}$. Hence, this study proposes to trace the profile of the research groups and publications focusing on nationwide food and nutrition-related interventions, diagnosis, or assessment in the context of the Family Health Strategy since 1994.

\section{METHODS}

\section{Structured review}

A Structured Review was conducted according to the systematic review principles of 
the Cochrane Collaboration, which uses predefined methods for identifying, selecting, and critically assessing studies, and collecting and analyzing data ${ }^{18}$. Systematic reviews should follow seven steps. The present review followed the first six listed below:

a) Formulating the question: What types of studies include food and nutrition actions in the context of Family Health Strategy?

b) Study search and selection: The searched databases were the Web of Science, Biblioteca Virtual em Saúde (BVS) (Lilacs, Medline, and SciELO) and Embase. Original studies conducted in Brazil from 1994 to December 2012 published in any language. The following keywords and their combinations were used for searching the title or abstract: in Portuguese, nutrição/alimentação; Atenção Primária à Saúde/Atenção Básica à Saúde; Estratégia Saúde da Família/Programa Saúde da Familia; and in English, nutrition/food; health primary care; family health. The selection of these terms relied on the health science descriptors available at the BVS, and similar terms in the other databases. The keywords used for the food and nutrition area were generic to enable the retrieval of journals with diverse characterizable actions.

c) Critical study assessment: The articles were initially selected by title and abstract, resulting in 151 articles. Next, the methodology was read to make sure the study met the inclusion criteria, namely studies in the Family Health Strategy context that approached food and nutrition actions. These are defined as a set of individual, family, and group actions at the primary care level of the health care system that aim to promote health, prevent and treat disease, and provide rehabilitation ${ }^{10}$ attributed to dieticians. These actions may also compose the actions answered by the health care team ${ }^{10}$.

Therefore, manuscripts that focused on other health-related themes were also selected, providing they had some specific or cross-sectional actions related to food and nutrition, such as infant care, infant growth and development monitoring; prenatal care monitoring weight gain; and references to the food intake and counseling, anthropometry, or adherence to nonpharmaceutical treatment of individuals with high blood pressure or diabetes.

Dissertations or theses were not included and the study journals should have at least a Qualis Capes classification of B3 for the area of Collective Health. Studies of populations not covered by the Family Health Strategy were not included, such as: studies based on samples of Primary Health Care Units Clientele, household census, traditional care, vaccination campaigns, polyclinics, Specialized Reference Units, Family Development Center, institutions, and projects linked to universities. The same exclusion criteria were used for studies of families covered only by the Pastoral da Criança, private health care insurance, or specific municipal programs such as the Programa de Suplementação Alimentar (Food Supplementation Program), and Programa do Desnutrido (Program for the Malnourished), among others. This further selection resulted in 44 articles that met the study criteria; another 10 articles were retrieved from their references section, totaling 54 studies.

d) Data collection: The following variables were collected: author, journal, and publication year; location; population, classified according to the municipality size (small, medium, large, and metropolis) provided by the Instituto Brasileiro de Geografia e Estatística (IBGE, Brazilian Institute of Geography and Statistics) ${ }^{19}$; Family Health Strategy presence in the municipality according to the Unified Health Care System database ${ }^{20}$; and Family Health Strategy coverage stratified by percentage brackets I - $0 \%$ to $20 \%$; II - 20\% to $50 \%, \mathrm{III}-50 \%$ to $70 \%, \mathrm{IV}->70 \%{ }^{21}$. Other study variables were the Municipal Human Development Indices (HDI-M)22, classified as medium (0.5 to 0.79 ) or high ( $\geq 0.80)$; study design, methods, sample size, and target population; study objective/theme; education of the first author retrieved from the Plataforma Lattes (Lattes Plataform) available at the Conselho Nacional de 
Desenvolvimento Científico e Tecnológico's (CNPq, National Council for Scientific and Technological Development) site ${ }^{23}$; the degree of nutritional intervention (diagnosis, health promotion, disease prevention, ambulatory care/treatment/care) or management (control, organization, planning, and supervision) according to the definition provided by the Ministry of Health's Matriz de Ações de Alimentação e Nutrição na Atenção Básica de Saúde (Matrix of Food and Nutrition Actions $)^{10}$.

e) Data analysis and presentation: the selected studies have different profiles and were grouped and classified according to their design, nutritional care action, and municipal management actions. These data are shown in tables.

f) Data interpretation: after result analysis and tabulation, we discussed the action profiles and applicability; food and nutrition in the context of Family Health Strategy in different types of Brazilian municipalities; the limitations and potentials of such services; and the human resources.

The seventh step regards improving and updating the manuscript after publication, which does not apply to the present study.

\section{Identification of the research groups that investigate about food and nutrition in the Family Health Strategy}

Conselho Nacional de Desenvolvimento Científico e Tecnológico's24, Diretórios dos Grupos de Pesquisa (DGP, Research Group Directory) search tools were used for finding the groups that work on this theme. The tools search not only the database but also census data (2000, 2002, 2004, 2006, 2008, and 2010). For both searches, the following search options were marked: group name, group repercussions, name of the research line, and keywords of the research line. The searched keywords were: collective health/public health; basic care/primary care; family health/ community agents together with the terms food/ nutrition (saúde coletiva/saúde pública, atenção básica/atenção primária, saúde da família/agentes comunitários, combinadas com os termos alimentação/nutrição). This procedure allowed searching the focus of this review more accurately, beyond the academic area that conceived the study, given that food and nutrition investigations may occur in other academic areas.

To complement the search in the above database, the groups were also searched using an alternative method. The full name of the authors of the articles selected in the structured review was used for finding other groups in which they worked as leaders or researchers.

The groups were selected by reading all the information provided on the group's web page and the objectives of one or more research lines. Studies on the following subjects were excluded: studies on animal food or nutrition, chemical composition of foods, clinical nutrition, food service management, and sports nutrition. When at least one of the research lines regarded the theme, the group was selected.

The following data were collected from the groups that studied primary care or Family Health Strategy with a focus on food and nutrition: year of establishment, predominant area, institution, research lines, and keywords.

\section{RE S U L T S}

\section{Research on food and nutrition in the Family Health Strategy}

Fifty-four original articles on some type of Family Health Strategy action related to food and nutrition were analyzed, two in English and all others in Portuguese. Although the search included all studies published since 1994, the 54 selected studies were published between 2002 and 2012, and most ( $n=40,74 \%$ ) were published in the last six years.

Only forty studies reported the data collection period, and these studies were mostly published from one to six years after completion 
of data collection. Only one study was published twelve years after completion of data collection.

The articles were found in twenty different journals, seventeen of which were Brazilian. Thirty-three $(61.0 \%)$ studies were published in public health, collective health, or epidemiology journals. Five were nursing journals, accounting for $24.1 \%$ of the publications ( $n=13)$. Only four studies were from two different nutrition journals, and another four studies were from specific medical journals.

Most studies were done in the Brazilian Southeast ( $n=27,50 \%)$, especially in the states of Minas Gerais and São Paulo. However, the other Brazilian regions were also represented: Northeast with eighteen studies (33\%), South with seven studies (13\%), Central-West with one study (2\%), and North with one study (2\%). A total of 44 municipalities were studied, and three studies included more than one municipality $\left(1^{\circ}=10 ; 2^{\circ}=4\right.$, and $\left.3^{\circ}=2\right)$. Two articles did not mention the study municipalities. The population in these municipalities varied from three thousand to ten million inhabitants. Seven municipalities were metropolis (>900 thousand inhabitants), eighteen were large (100 thousand to 900 thousand inhabitants), two were medium-sized (50 thousand to 100 thousand inhabitants), and seventeen were small, with less than 20 thousand inhabitants.

The municipalities' Human Development Index (HDI-M) ranged from medium (0.500-0.799, $\mathrm{n}=34)$ to high $(\geq 0.800, \mathrm{n}=10)^{22}$. According to Datasus, 31 municipalities had Family Health Strategy coverage higher than 50\%; and of these, 20 had Family Health Strategy coverage higher than $70 \%{ }^{20}$.

The academic education of the first author of the reviewed studies included: dieticians with 16 studies; nurses with 14 studies; physicians with 12 studies; psychologists with 3 studies; physical therapists, dental surgeons, and physical educators with 2 studies each; biologist, occupational therapist, and pharmacist with one study each.
Moststudies focused on a specific population, such as children $(n=14)$, health professionals $(n=12)$, older adults $(n=5)$, pregnant women $(n=3)$, mothers $(n=3)$, adults $(n=2)$, and women of childbearing age $(n=2)$. Some studies included more than one type of population, such as adults and older adults $(n=8)$, mothers and children $(n=2)$, mothers and professionals $(n=1)$, and professionals and pregnant women $(n=1)$. One study focused on a child's father.

Some themes can be categorized according to the articles' objectives and the matrix of food and nutrition actions in primary care ${ }^{10}$. High blood pressure or diabetes mellitus was the most common topic $(n=12,22.2 \%)$, followed by breastfeeding $(n=11)$, nutritional status assessment (anthropometry, $n=9$ ), child health or childcare $(n=7)$, prenatal care $(n=6)$, micronutrient inadequacies $(n=5)$, labor $(n=2)$, welfare program $(n=1)$, and general food and nutrition $(n=1)$. Articles about the Sistema de Vigilância Alimentar e Nutricional (Sisvan, Food and Nutrition Surveillance System) were not found.

The methods used in the study articles varied: structured or semi-structured interviews with users or professionals; self-administered questionnaires; venous or finger-stick blood tests; stool test; direct anthropometry; direct blood pressure measurement; food intake assessment; field diary; direct observation of health unit structure; and use of scales and tests to measure child development and older adult motor development. The interventions included: iron supplementation; physical activity; Family Health Strategy-related; provision of individual, group, and home care; a strategy called Atenção Integrada às Doenças Prevalentes na Infância (AIDPI, Integrated Management of Childhood IIIness); and Breastfeeding - Friendly Primary and Prenatal Care Units. The educational intervention proposals used dialogue discourse, demonstration, service practices, lectures, group dynamics, and case studies.

Tables 1, 2, and 3 show the studies. Table 1 lists quantitative or mostly quantitative studies 
Table 1. Profile of the quantitative or mostly quantitative studies on disease diagnosis and prevention, and health promotion and care. Brazil, 2003 to 2012.

\begin{tabular}{|c|c|c|c|c|c|c|c|c|}
\hline Author, year & $\begin{array}{l}\text { Municípalities } \\
\text { (n), Region }\end{array}$ & Size $^{a}$ & FHS cover ${ }^{\mathbf{b}}$ & $\mathrm{HDI}-\mathrm{M}^{\mathrm{c}}$ & Sample & Topic & Purpose $^{d}$ & $\begin{array}{l}\text { Education of } \\
\text { the } 1^{\circ} \text { author }\end{array}$ \\
\hline $\begin{array}{l}\text { Borges \& } \\
\text { Philippi }(2003)^{25}\end{array}$ & (1), SE & M & $\|$ & High & 41 lactating women & Breastfeeding & $\mathrm{D}$ & Nurse \\
\hline $\begin{array}{l}\text { Ferreira et al. } \\
(2003)^{26}\end{array}$ & (1), NE & $\mathrm{L}$ & III & Medium & $\begin{array}{l}293 \text { children aged } 6 \\
\text { to } 23 \text { months }\end{array}$ & $\begin{array}{l}\text { Micronutrient } \\
\text { inadequacy }\end{array}$ & DP & Nurse \\
\hline $\begin{array}{l}\text { Sousa \& Araújo } \\
(2004)^{27}\end{array}$ & (1), NE & L & III & Medium & $\begin{array}{l}371 \text { children aged } 6 \\
\text { to } 60 \text { months }\end{array}$ & $\begin{array}{l}\text { Micronutrient } \\
\text { inadequacy }\end{array}$ & D & Nurse \\
\hline $\begin{array}{l}\text { Mano \& Pierin } \\
(2005)^{28}\end{array}$ & (1), SE & M & $\|$ & High & $\begin{array}{l}226 \text { records of } \\
\text { hypertensives in the } \\
\text { FHS or not }\end{array}$ & HBP/DM & D & Nurse \\
\hline $\begin{array}{l}\text { Parada et al. } \\
(2005)^{29}\end{array}$ & (1), SE & $S$ & IV & Medium & $\begin{array}{l}166 \text { children aged } \\
<1 \text { year }\end{array}$ & Breastfeeding & D & Nurse \\
\hline $\begin{array}{l}\text { Barbosa et al. } \\
(2006)^{30}\end{array}$ & (1), NE & L & IV & Medium & 284 older adults & $\begin{array}{l}\text { Micronutrient } \\
\text { inadequacy }\end{array}$ & D & Dietician \\
\hline $\begin{array}{l}\text { Maria-Mengel \& } \\
\text { Linhares (2007) }\end{array}$ & (1), SE & L & I & High & $\begin{array}{l}120 \text { children aged } 6 \\
\text { to } 44 \text { months }\end{array}$ & $\begin{array}{l}\text { Child health/ } \\
\text { care }\end{array}$ & D & Psychologist \\
\hline $\begin{array}{l}\text { Melo et al. } \\
(2007)^{32}\end{array}$ & (1), NE & L & $\|$ & Medium & $\begin{array}{l}115 \text { pregnant } \\
\text { women }\end{array}$ & $\begin{array}{l}\text { Nutritional } \\
\text { status }\end{array}$ & D & Physician \\
\hline $\begin{array}{l}\text { Nascimento et } \\
\text { al. }(2007)^{33}\end{array}$ & (1), NE & L & IV & Medium & 315 older adults & $\begin{array}{l}\text { Micronutrient } \\
\text { inadequacy }\end{array}$ & D & Dietician \\
\hline $\begin{array}{l}\text { Alves et al. } \\
(2008)^{34}\end{array}$ & (1), NE & M & III & Medium & $\begin{array}{l}68 \text { children aged } 5 \\
\text { to } 10 \text { years with } \\
\mathrm{BMI} \geq 85^{\text {th }}\end{array}$ & $\begin{array}{l}\text { Nutritional } \\
\text { status }\end{array}$ & DP & Physician \\
\hline $\begin{array}{l}\text { Azeredo et al. } \\
(2008)^{35}\end{array}$ & (1), SE & $S$ & IV & Medium & $\begin{array}{l}36 \text { professionals } \\
\text { and } 137 \text { mothers of } \\
\text { children aged }<24 \\
\text { months }\end{array}$ & Breastfeeding & D & Dietician \\
\hline $\begin{array}{l}\text { Assunção \& } \\
\text { Ursine }(2008)^{36}\end{array}$ & (1), SE & M & III & High & $\begin{array}{l}164 \text { diabetics aged } \\
>18 \text { years }\end{array}$ & HBP/DM & NCT & $\begin{array}{l}\text { Physical } \\
\text { therapist }\end{array}$ \\
\hline $\begin{array}{l}\text { Cotta et al. } \\
(2009)^{37}\end{array}$ & (1), SE & s & IV & Medium & $\begin{array}{l}150 \text { hypertensives, } \\
5 \text { diabetics, and } 25 \\
\text { with DM and HBP }\end{array}$ & HBP/DM & D & $\begin{array}{l}\text { Occupational } \\
\text { therapist }\end{array}$ \\
\hline $\begin{array}{l}\text { Girotto et al. } \\
(2009)^{38}\end{array}$ & (1), S & L & III & High & $\begin{array}{l}385 \text { hypertensive } \\
\text { adults and older } \\
\text { adults }\end{array}$ & HBP/DM & D & Pharmacist \\
\hline $\begin{array}{l}\text { Azeredo et al. } \\
(2010)^{39}\end{array}$ & (1), SE & MS & III & High & $\begin{array}{l}103 \text { anemic } \\
\text { children }\end{array}$ & $\begin{array}{l}\text { Micronutrient } \\
\text { inadequacy }\end{array}$ & DP & Dietician \\
\hline $\begin{array}{l}\text { Brecailo et al. } \\
(2010)^{40}\end{array}$ & (1), S & L & III & Medium & $\begin{array}{l}426 \text { children aged } \\
<2 \text { years }\end{array}$ & Breastfeeding & D & Dietician \\
\hline $\begin{array}{l}\text { Felisbino- } \\
\text { Mendes et al. } \\
(2010)^{41}\end{array}$ & (1), SE & $S$ & IV & Medium & $\begin{array}{l}1332 \text { children aged } \\
<10 \text { years }\end{array}$ & $\begin{array}{l}\text { Nutritional } \\
\text { status }\end{array}$ & D & Nurse \\
\hline $\begin{array}{l}\text { Silva et al. } \\
(2010)^{42}\end{array}$ & (1), SE & L & I & High & $\begin{array}{l}43 \text { diabetic older } \\
\text { adults }\end{array}$ & HBP/DM & D & Nurse \\
\hline $\begin{array}{l}\text { Cristovão et al. } \\
(2011)^{43}\end{array}$ & (1), SE & M & $\|$ & High & 298 adult women & $\begin{array}{l}\text { Nutritional } \\
\text { status }\end{array}$ & D & Nurse \\
\hline $\begin{array}{l}\text { Garcia et al. } \\
(2011)^{44}\end{array}$ & (1), N & $S$ & IV & Medium & $\begin{array}{l}164 \text { children aged } 6 \\
\text { to } 24 \text { months }\end{array}$ & $\begin{array}{l}\text { Nutritional } \\
\text { status }\end{array}$ & D & Dietician \\
\hline
\end{tabular}


Table 1. Profile of the quantitative or mostly quantitative studies on disease diagnosis and prevention, and health promotion and care. Brazil, 2003 to 2012.

\begin{tabular}{|c|c|c|c|c|c|c|c|c|}
\hline Author, year & $\begin{array}{l}\text { Municípalities } \\
\text { (n), Region }\end{array}$ & Size $^{a}$ & FHS cover ${ }^{\mathbf{b}}$ & $\mathrm{HDI}-\mathrm{M}^{c}$ & Sample & Topic & Purpose $^{d}$ & $\begin{array}{l}\text { Education of } \\
\text { the } 1^{\circ} \text { author }\end{array}$ \\
\hline $\begin{array}{l}\text { Cunha et al. } \\
(2012)^{45}\end{array}$ & (1), CW & $S$ & IV & Medium & $\begin{array}{l}80 \text { enrolled in the } \\
\text { HiperDia }\end{array}$ & HBP/DM & D & $\begin{array}{l}\text { Physical } \\
\text { educator/ } \\
\text { therapist }\end{array}$ \\
\hline $\begin{array}{l}\text { Marinho et al. } \\
(2012)^{46}\end{array}$ & (1), NE & L & IV & Medium & $\begin{array}{l}419 \text { non-diabetic } \\
\text { adults }\end{array}$ & $\begin{array}{l}\text { Nutritional } \\
\text { status }\end{array}$ & D & Nurse \\
\hline $\begin{array}{l}\text { Ribeiro et al. } \\
(2012)^{47}\end{array}$ & (1), SE & $S$ & IV & Medium & $\begin{array}{l}27 \text { hypertensive } \\
\text { women }\end{array}$ & HBP & HP & Dietician \\
\hline $\begin{array}{l}\text { Soares et al. } \\
(2012)^{48}\end{array}$ & (1), NE & L & III & Medium & 235 older adults & $\begin{array}{l}\text { Nutritional } \\
\text { status }\end{array}$ & D & $\begin{array}{l}\text { Physical } \\
\text { therapist }\end{array}$ \\
\hline $\begin{array}{l}\text { Ferreira-Marim } \\
\text { et al. }(2012)^{49}\end{array}$ & (1), SE & L & I & High & $\begin{array}{l}155 \text { preschoolers } \\
\text { aged } 2 \text { to } 5 \text { years }\end{array}$ & $\begin{array}{l}\text { Nutritional } \\
\text { status }\end{array}$ & D & Dietician \\
\hline $\begin{array}{l}\text { Vianna et al. } \\
(2012)^{50}\end{array}$ & (1), SE & $S$ & IV & Medium & 70 older adults & $\begin{array}{l}\text { Nutritional } \\
\text { status }\end{array}$ & DP & $\begin{array}{l}\text { Physical } \\
\text { educator }\end{array}$ \\
\hline
\end{tabular}

Note: aSize of the municipality. Source: Instituto Brasileiro de Geografia e Estatística, 200719; S: Small; MS: Medium-Sized; L: Large, M: Metropolis; bSource: DATASUS, 2007; FHS cover: Family Health Strategy coverage as follows: I - 0\% to 20\%; II - 20 to 50\%, III - 50 to $70 \%$, IV - >70\%; cSource: Programa das Nações Unidas para o Desenvolvimento, 2000. HDI-M: Human Development Index of the Municipality classified as medium (0.5 to 0.799 ) and high ( $\geq 0.80)$; ${ }^{d}$ Focus of the food and nutrition actions according to the source Brasil, 2009.

D: Diagnosis; HP: Health Promotion; DP: Disease Prevention; NCT: Nutritional Ambulatory Care/Care/Treatment; HBP: High Blood Pressure; DM: Diabetes Mellitus; SE: Southeast; NE: Northeast; CW: Central West; S: South; N: North.

that performed nutritional care actions, such as diagnosis, health promotion, disease prevention, and ambulatory care/care/treatment. Table 2 also lists quantitative studies, but only those that performed food and nutrition actions typical of local or municipal management. Table 3 shows the qualitative studies and the actions include both nutritional care and management.

Forty-two quantitative studies were found, two mostly quantitative with some qualitative data. Of these, 26 referred to nutritional care actions (Table 1) and 16 to local or municipal management actions (Table 2).

The studies in Table 1 are either interventional $(n=5)$, cross-sectional $(n=20)$, or cohort $(n=1)$. The five intervention studies assessed effectiveness $26,34,39,47,50$.

Three articles with retrospective information involved the organization of secondary data, one of which combined primary and secondary data. These articles collected data from medical records and health information systems, namely from the
Sistema de Informação da Atenção Básica (SIAB, Primary Care Information System).

Thirteen studies in Table 1 studied locationspecific populations, such as subjects living in a district or users of a health service, and another thirteen studies regarded other types of specific populations, such as a sample of Family Health Strategy professionals representative of the municipal Family Health Strategy professional population.

Most quantitative studies $(n=20)$ used nutritional status diagnosis (intake assessment, anthropometric assessment, breastfeeding duration, micronutrient deficiencies) for assessing nutritional care actions. Although mostly quantitative, two studies also presented psychosocial and cultural data (perceived situations, professional practices, and cultural practices). Four investigated disease-prevention methods (prophylactic iron supplementation and practice of physical activity in older adults or obese children), one promoted health (nutritional education for hypertensives), 
Table 2. Profile of the quantitative studies that approach food and nutrition actions promoted by municipal or local administration. Brazil, 2002 to 2012.

\begin{tabular}{|c|c|c|c|c|c|c|c|c|}
\hline Author, year & Municípalities & Size $^{a}$ & FHS cover ${ }^{\mathbf{b}}$ & HDI-Mc & Sample & Topic & Purpose $^{d}$ & $\begin{array}{l}\text { Education of } \\
\text { the } 1^{\circ} \text { author }\end{array}$ \\
\hline $\begin{array}{l}\text { Cesar et al. } \\
(2002)^{51}\end{array}$ & (2), SE & 25 & $2 \mathrm{IV}$ & 2 Medium & 409 children & $\begin{array}{l}\text { Child health/ } \\
\text { care }\end{array}$ & control & Physician \\
\hline $\begin{array}{l}\text { Ciconi et al. } \\
(2004)^{52}\end{array}$ & (1), SE & L & $\|$ & Medium & 61 professionals & Breastfeeding & organization & Biologist \\
\hline $\begin{array}{l}\text { Dubeux et al. } \\
(2004)^{53}\end{array}$ & (1), NE & L & $\|$ & Medium & 40 teams & Breastfeeding & control & $\begin{array}{l}\text { Dental } \\
\text { surgeon }\end{array}$ \\
\hline $\begin{array}{l}\text { Faleiros et al. } \\
(2005)^{54}\end{array}$ & (1), $S$ & L & $\|$ & High & 112 children & Breastfeeding & control & Physician \\
\hline $\begin{array}{l}\text { Roncalli \& Lima } \\
(2006)^{55}\end{array}$ & (4), NE & - & - & - & $\begin{array}{l}2144 \text { children < } 5 \\
\text { years w/ and w/o } \\
\text { FHS }\end{array}$ & $\begin{array}{l}\text { Child health/ } \\
\text { care }\end{array}$ & control & $\begin{array}{l}\text { Dental } \\
\text { surgeon }\end{array}$ \\
\hline $\begin{array}{l}\text { Araújo \& } \\
\text { Guimarães } \\
(2007)^{56}\end{array}$ & (1), NE & $M$ & I & High & $\begin{array}{l}135 \text { adults/ older } \\
\text { adults with HBP }\end{array}$ & HBP/DM & control & Physician \\
\hline $\begin{array}{l}\text { Caldeira et al. } \\
(2007)^{57}\end{array}$ & (1), SE & L & $\|$ & Medium & $\begin{array}{l}\text { Professionals: } 41 \\
\text { with higher } \\
\text { education and } 152 \\
\text { with high school }\end{array}$ & Breastfeeding & $\begin{array}{l}\text { organization } \\
\text { and control }\end{array}$ & Physician \\
\hline $\begin{array}{l}\text { Lima et al. } \\
(2007)^{58}\end{array}$ & (1), SE & $S$ & IV & Medium & $\begin{array}{l}49 \text { postpartum } \\
\text { women and } 26 \\
\text { newborns }\end{array}$ & $\begin{array}{l}\text { Child health/ } \\
\text { care }\end{array}$ & control & Nurse \\
\hline $\begin{array}{l}\text { Caldeira et al. } \\
(2008)^{59}\end{array}$ & (1), SE & L & $\|$ & Medium & $\begin{array}{l}\text { About } 1400 \\
\text { mothers }\end{array}$ & Breastfeeding & organization & Physician \\
\hline $\begin{array}{l}\text { Cesar et al. } \\
(2008)^{60}\end{array}$ & (1), $S$ & L & $\|$ & Medium & $\begin{array}{l}361 \text { pregnant } \\
\text { women }\end{array}$ & Prenatal care & control & Physician \\
\hline $\begin{array}{l}\text { Costa et al. } \\
(2009)^{61}\end{array}$ & (1), SE & S & IV & Medium & $\begin{array}{l}33 \text { pregnant } \\
\text { women and } 35 \\
\text { professionals }\end{array}$ & Prenatal care & $\begin{array}{l}\text { organization } \\
\text { and control }\end{array}$ & Dietician \\
\hline $\begin{array}{l}\text { Lima et al. } \\
(2009)^{62}\end{array}$ & (1), SE & M & I & High & 472 hypertensives & HBP/DM & $\begin{array}{l}\text { planning } \\
\text { and control }\end{array}$ & Physician \\
\hline $\begin{array}{l}\text { Niquini et al. } \\
(2010)^{63}\end{array}$ & (1), SE & M & I & High & $\begin{array}{l}7 \mathrm{FHCU} \text { e } 230 \\
\text { pregnant women }\end{array}$ & Prenatal care & organization & Dietician \\
\hline $\begin{array}{l}\text { Niquini et al. } \\
(2012)^{64}\end{array}$ & (1), SE & $M$ & I & High & $\begin{array}{l}230 \text { pregnant } \\
\text { women }\end{array}$ & Prenatal care & control & Dietician \\
\hline $\begin{array}{l}\text { Cervato- } \\
\text { Mancuso et al. } \\
(2012)^{65}\end{array}$ & (1), SE & M & $\|$ & High & $\begin{array}{l}123 \text { dieticians from } \\
\text { primary care and } 51 \\
\text { from NASF }\end{array}$ & $\begin{array}{l}\text { Professional } \\
\text { practice }\end{array}$ & $\begin{array}{l}\text { planning } \\
\text { and } \\
\text { organization }\end{array}$ & Dietician \\
\hline $\begin{array}{l}\text { Cesar et al. } \\
(2012)^{66}\end{array}$ & (1), $S$ & L & $\|$ & Medium & 2395 mothers & Prenatal care & control & Physician \\
\hline
\end{tabular}

Note: asize of the municipality. Source: Instituto Brasileiro de Geografia e Estatística, 2007¹9; S: Small; MS: Medium-Sized; L: Large, M: Metropolis; bSource: DATASUS, 2007; FHS cover: Family Health Strategy coverage as follows: I - 0\% to 20\%; II - 20 to 50\%, III - 50 to $70 \%$, IV - >70\%; cSource: Programa das Nações Unidas para o Desenvolvimento, 2000. HDI-M: Human Development Index of the Municipality classified as medium (0.5 to 0.799) and high ( $\geq 0.80)$; ${ }^{d}$ The local/municipal management consists of the following elements: planning, organization, management, and control. FHCU: Family Health Care Unit; NASF: Family Health Support Center; HBP: High Blood Pressure; DM: Diabetes Mellitus; SE: Southeast; NE: Northeast; CW: Central West; S: South; N: North. 
Table 3. Characterization of the qualitative studies on diagnosis, health promotion/care, and management. Brazil, 2003 to 2012.

\begin{tabular}{|c|c|c|c|c|c|c|c|c|}
\hline Author, year & Municípalities & $\operatorname{Size}^{a}$ & FHS cover ${ }^{\mathbf{b}}$ & $\mathrm{HDI}-\mathrm{M}^{\mathrm{c}}$ & Sample & Topic & Purpose $^{d}$ & $\begin{array}{l}\text { Education of } \\
\text { the } 1^{\circ} \text { author }\end{array}$ \\
\hline $\begin{array}{l}\text { Felisberto et al. } \\
(2002)^{67}\end{array}$ & (10), NE & $\begin{array}{l}6 \mathrm{~S} ; 1 \\
\mathrm{MS} ; \\
3 \mathrm{~L}\end{array}$ & $\begin{array}{l}2 \text { III } \\
\text { and } 8 \\
\text { IV }\end{array}$ & $\begin{array}{l}10 \\
\text { Medium }\end{array}$ & $\begin{array}{l}10 \text { coordinators } \\
\text { and } 146 \\
\text { professionals }\end{array}$ & $\begin{array}{l}\text { Child health/ } \\
\text { care }\end{array}$ & $\begin{array}{l}\text { MT - } \\
\text { control }\end{array}$ & Physician \\
\hline $\begin{array}{l}\text { Fernandez et al. } \\
(2005)^{68}\end{array}$ & (1), SE & M & $\|$ & High & $\begin{array}{l}8 \text { physicians, } 8 \\
\text { nurses, } 45 \text { CA, } 16 \\
\text { nurse assistants }\end{array}$ & $\begin{array}{l}\text { Food and } \\
\text { nutrition }\end{array}$ & $\begin{array}{l}\text { MT - } \\
\text { organization }\end{array}$ & Dietician \\
\hline $\begin{array}{l}\text { Alves e Nunes } \\
(2006)^{69}\end{array}$ & (3), NE & - & - & - & $\begin{array}{l}50 \text { appointments } \\
\text { with } 10 \mathrm{FHS} \\
\text { physicians }\end{array}$ & HBP/DM & $\mathrm{HP}$ & Psychologist \\
\hline $\begin{array}{l}\text { Bustamante e } \\
\text { Trad }(2007)^{70}\end{array}$ & (1), NE & M & I & High & $\begin{array}{l}6 \text { families with } \\
\text { small children }\end{array}$ & $\begin{array}{l}\text { Child health/ } \\
\text { care }\end{array}$ & D & Psychologist \\
\hline $\begin{array}{l}\text { Slomp et al. } \\
(2007)^{71}\end{array}$ & (1), S & L & III & Medium & 90 newborns & $\begin{array}{l}\text { Child health/ } \\
\text { care }\end{array}$ & NCT & Nurse \\
\hline $\begin{array}{l}\text { Frota et al. } \\
(2009)^{72}\end{array}$ & (1), NE & S & IV & Medium & $\begin{array}{l}15 \text { mothers with } \\
\text { children aged }<1 \\
\text { year }\end{array}$ & Breastfeeding & $\mathrm{D}$ & Nurse \\
\hline $\begin{array}{l}\text { Pontes et al. } \\
(2009)^{73}\end{array}$ & (1), NE & M & III & Medium & $\begin{array}{l}17 \text { couples and } \\
\text { parents of children } \\
\text { aged } 6 \text { to } 8 \text { months }\end{array}$ & Breastfeeding & $\mathrm{D}$ & Nurse \\
\hline $\begin{array}{l}\text { Silva et al. } \\
(2009)^{74}\end{array}$ & (1), SE & L & $\|$ & Medium & 15 physicians & $\begin{array}{l}\text { Prenatal/child } \\
\text { care }\end{array}$ & NCT & Physician \\
\hline $\begin{array}{l}\text { Araújo et al. } \\
(2010)^{75}\end{array}$ & (1), NE & $S$ & IV & Medium & 11 nurses & HBP/DM & NCT & Nurse \\
\hline $\begin{array}{l}\text { Pinto \& Bosi } \\
(2010)^{76}\end{array}$ & (1), NE & $M$ & I & Medium & $\begin{array}{l}8 \text { obese adult } \\
\text { females }\end{array}$ & $\begin{array}{l}\text { Nutritional } \\
\text { status }\end{array}$ & D & Dietician \\
\hline $\begin{array}{l}\text { Ramos \& Cuervo } \\
(2012)^{77}\end{array}$ & (1), S & M & I & High & $\begin{array}{l}11 \mathrm{PHCU} \text { or } \mathrm{FHCU} \\
\text { coordinators and } \\
12 \text { professionals }\end{array}$ & $\begin{array}{l}\text { Welfare } \\
\text { program/HRAF }\end{array}$ & $\begin{array}{l}\text { MT - } \\
\text { planning } \\
\text { and } \\
\text { organization }\end{array}$ & Dietician \\
\hline $\begin{array}{l}\text { Camossa et al. } \\
(2012)^{78}\end{array}$ & (1), SE & $L$ & $\|$ & High & $\begin{array}{l}21 \text { professionals } \\
\text { from } 2 \text { teams, } 6 \\
\text { residents and } 1 \\
\text { manager }\end{array}$ & $\begin{array}{l}\text { Professional } \\
\text { practice }\end{array}$ & $\begin{array}{l}\text { MT - } \\
\text { planning } \\
\text { and } \\
\text { organization }\end{array}$ & Dietician \\
\hline
\end{tabular}

Note: asize of the municipality. Source: Instituto Brasileiro de Geografia e Estatística, 200719; S: Small; MS: Medium-Sized; L: Large, M: Metropolis; bSource: DATASUS, 2007; FHS cover: Family Health Strategy coverage as follows: I - 0\% to 20\%; II - 20\% to 50\%, III - 50\% to 70\%, IV - >70\%; 'Source: Programa das Nações Unidas para o Desenvolvimento, 2000. HDI-M: Human Development Index of the Municipality classified as medium (0.5 to 0.799) and high ( $\geq 0.80)$; ${ }^{\mathrm{d}}$ Focus of the food and nutrition actions according to the source Brasil, 2009.

D: Diagnosis; HP: Health Promotion; DP: Disease Prevention; NCT: Nutritional Ambulatory Care/Care/Treatment; The local/Municipal Management (MT) consists of the following elements: planning, organization, management, and control; HBP: High Blood Pressure; DM: Diabetes Mellitus; PHCU: Primary Health Care Unit; FHCU: Family Health Care Unit; HRAF: Human Right to Adequate Food; CA: Community Agents; SE: Southeast; NE: Northeast; CW: Central West; S: South; N: North.

and one provided ambulatory care/care/treatment (assistance to diabetics).

Table 2 shows studies with managementattribution actions. Sixteen studies were quantitative, of which four were intervention studies, ten were cross-sectional studies, one was a cohort study, and one was a community-based trial study. Five of these studies regarded secondary data (two were retrospective) obtained from the child health booklet (which contains a child's health, vaccination, and nutritional data called Cardeneta da Criança), medical records, 
national health establishment registry, Municipal Health Department and pregnant women's record (Cartão da Gestante).

Most studies on management $(n=15)$ consisted of assessments: five studies made impact assessments ${ }^{51,54-56,60}$; one assessed effectiveness ${ }^{59}$; nine made service assessments, of which one analyzed structure, process, and result ${ }^{61}$; three studies approached only two of these dimensions ${ }^{53,57,62}$; and five assessed only one of these dimensions $s^{52,58,63-65}$. The only study that did not involve an assessment analyzed the dietician's work in a municipality, and whether the municipality had an adequate number of dieticians ${ }^{66}$.

Eleven studies included samples representative of the municipality ${ }^{51-53,57,59-61,63-66}$, four included specific samples (Health Care Center or Sanitation District) $)^{54,56,58,62}$, and one with regional representativeness included samples of four municipalities of two Brazilian Northeast states $^{55}$.
Regarding management actions (Table 2), nine publications regarded control; three regarded specific organization actions; two regarded organization and control actions; and two regarded planning and control actions.

Table 3 shows the twelve qualitative studies that combined different nutritional care and management actions. Despite their qualitative nature, two had representative samples: the first included coordinators and professionals from ten municipalities in a Brazilian-Northeast state ${ }^{67}$; and the second included physicians from a municipality ${ }^{74}$.

Few studies in this table consisted of assessments. One study assessed structure ${ }^{68}$; two assessed process ${ }^{69,77}$, and one assessed structure and process ${ }^{67}$.

One of these studies used secondary data sources, namely birth certificates and medical records ${ }^{71}$.

Some articles of the tables 1, 2, and 3 analyzed when the Family Health Strategy was

Table 4. Distribution of the research groups by keywords. Brazil, 2000-2012.

\begin{tabular}{|c|c|c|c|c|c|c|c|}
\hline Keywords & 2000 & 2002 & 2004 & 2006 & 2008 & 2010 & Database* \\
\hline $\mathrm{CH}+\mathrm{Nut}$ & 7 & 19 & 24 & 24 & 21 & 34 & 42 \\
\hline $\mathrm{CH}+$ Diet & 0 & 2 & 9 & 11 & 10 & 18 & 27 \\
\hline $\mathrm{CH}+\mathrm{Nut}+$ Diet & 0 & 2 & 8 & 11 & 9 & 18 & 27 \\
\hline $\mathrm{PH}+\mathrm{Nut}$ & 6 & 16 & 23 & 26 & 27 & 33 & 35 \\
\hline $\mathrm{PH}+$ Diet & 0 & 6 & 10 & 12 & 12 & 14 & 21 \\
\hline $\mathrm{PH}+\mathrm{Nut}+$ Diet & 0 & 5 & 9 & 10 & 10 & 12 & 19 \\
\hline $\mathrm{BC}+\mathrm{Nut}$ & 0 & 1 & 2 & 2 & 6 & 8 & 9 \\
\hline $\mathrm{BC}+$ Diet & 0 & 1 & 2 & 2 & 5 & 6 & 5 \\
\hline $\mathrm{BC}+\mathrm{Nut}+$ Diet & 0 & 1 & 2 & 2 & 4 & 5 & 5 \\
\hline $\mathrm{PC}+\mathrm{Nut}$ & 0 & 2 & 2 & 1 & 1 & 5 & 10 \\
\hline PC + Diet & 0 & 2 & 2 & 4 & 3 & 5 & 10 \\
\hline $\mathrm{PC}+\mathrm{Nut}+$ Diet & 0 & 0 & 0 & 1 & 1 & 3 & 7 \\
\hline $\mathrm{FHS}+\mathrm{Nut}$ & 2 & 3 & 4 & 9 & 11 & 11 & 10 \\
\hline FHS + Diet & 0 & 1 & 1 & 6 & 8 & 6 & 7 \\
\hline $\mathrm{FHS}+\mathrm{Nut}+$ Diet & 0 & 0 & 0 & 3 & 5 & 4 & 5 \\
\hline $\mathrm{CA}+\mathrm{Nut}$ & 0 & 0 & 1 & 1 & 1 & 1 & 1 \\
\hline$C A+$ Diet & 0 & 0 & 0 & 0 & 0 & 0 & 1 \\
\hline $\mathrm{CA}+\mathrm{Nut}+$ Diet & 0 & 0 & 0 & 0 & 0 & 0 & 1 \\
\hline
\end{tabular}

Note: "number of research groups found in the database during the search period. Nut: Nutrition (Nutrição); Diet: Diet (Alimentação); CH: Collective Health (Saúde Coletiva); PH: Public Health (Saúde Pública); BA: Basic Care (Atenção Básica); PC: Primary Care (Atenção Primária); FHS: Family Health Strategy (Estratégia Saúde da Família); CA: Community Agents (Agentes Comunitários). 
implemented in the study municipalities: 199735,39, $1998^{74}, 2000^{29,52}, 2001^{51,70}$, and 2002 40,54,62.

\section{Research groups}

Table 4 shows the distribution of the research groups according to the RGD from 2000 on and the great increase in the area's research capacity. The groups who explicitly cited primary care as a study field of food and nutrition numbered two in 2000, eight in 2002, eleven in 2004, 18 in 2006, 22 in 2008, 24 in 2010, and 26 when the database was consulted. Some groups focused at least one research line in food and nutrition within the Family Health Strategy: one in 2002, two in 2004, three in 2006, four in 2008, seven in 2010, and four in the database.

On the other hand, the search based on tracking the authors' names found another 30 groups that studied food and nutrition in primary care, totaling 54 groups when added to the groups found by keywords, and only two more groups focusing on the Family Health Strategy, totaling five research groups. The research lines of these groups regarding primary care and Family Health Strategy totaled 82 and 8, respectively.

Table 5 shows the characteristics of the research groups found in the database by keywords and author tracking. The year of establishment of the groups that focused on primary care varied considerably, but these groups became more common after 2000. Meanwhile, the groups that focused on Family Health Strategy appeared somewhat recently.

Most groups' institutions are located in the Brazilian Southeast $(n=25)$, Northeast $(n=16)$, and South $(n=11)$ regions, and most of the groups were from the areas of nutrition $(n=25)$, collective/ public health $(n=18)$, and medicine $(n=11)$.

Table 5. Distribution of the research groups during the search period according to year of establishment, institution location by Brazilian region, and prevailing academic area. Brazil, 2012.

\begin{tabular}{|c|c|c|c|c|c|c|c|c|}
\hline \multirow[b]{3}{*}{ Base corrente } & \multicolumn{2}{|c|}{ Year of establishment (n) } & \multicolumn{2}{|c|}{ Brazilian region of the institution } & \multicolumn{4}{|c|}{ Prevailing academic area } \\
\hline & \multirow{2}{*}{$\frac{\text { PHC }}{1976(1)}$} & \multirow{2}{*}{$\frac{\text { FHS }}{1999(1)}$} & $\mathrm{PHC}$ & FHS & \multicolumn{2}{|l|}{$\mathrm{PHC}$} & \multicolumn{2}{|c|}{ FHS } \\
\hline & & & SE (22) & SE (3) & Nutrition & (23) & Collective & $h(2)$ \\
\hline & $1982(1)$ & $2002(1)$ & NE (15) & $C W(1)$ & Collective healt & $h(16)$ & Nutrition & (2) \\
\hline & $1989(1)$ & $2007(1)$ & $S \quad(11)$ & NE (1) & Medicine & $(10)$ & Medicine & (1) \\
\hline & $1990(2)$ & $2009(2)$ & CW (5) & & Others & (5) & & \\
\hline & $1992(2)$ & & $N \quad(1)$ & & & & & \\
\hline & $1995(1)$ & & & & & & & \\
\hline & $1996(1)$ & & & & & & & \\
\hline & $1997(1)$ & & & & & & & \\
\hline & $1999(1)$ & & & & & & & \\
\hline & $2000(7)$ & & & & & & & \\
\hline & $2001(1)$ & & & & & & & \\
\hline & $2002(1)$ & & & & & & & \\
\hline & $2004(6)$ & & & & & & & \\
\hline & $2005(3)$ & & & & & & & \\
\hline & $2006(5)$ & & & & & & & \\
\hline & $2007(2)$ & & & & & & & \\
\hline & $2008(3)$ & & & & & & & \\
\hline & $2009(2)$ & & & & & & & \\
\hline & $2010(4)$ & & & & & & & \\
\hline & $2011(5)$ & & & & & & & \\
\hline & $2012(4)$ & & & & & & & \\
\hline Total & 54 & 5 & 54 & 5 & 54 & & 5 & \\
\hline
\end{tabular}

Note: PHC: Primary Health Care; FHS: Family Health Strategy; SE: Southeast; NE: Northeast; CW: Central West; S: South; N: North. 


\section{DISCUSSION}

The present results indicated a scarcity of studies on food and nutrition within the scope of the Family Health Strategy. However, other studies may exist since this review aimed to search only in the renowned journals. It is also possible that the Family Health Strategy management in some municipalities conducts only routine diagnoses and assessments, published in technical, not scientific, publications.

The Family Health Strategy was implemented in the municipalities from four to eight years after the agreement between the Ministry of Health and the states and municipalities for the construction of a new model (1994). Family Health Strategy coverage also varied greatly, from $14.5 \%$ to $94.0 \%$ in the referenced municipalities. Yet, high coverage does not ensure complete implementation of all the actions related to primary care's basic strategy areas ${ }^{79}$.

Family Health Strategy coverage is increasing much more rapidly in small Brazilian municipalities than in medium-sized and large municipalities. The advantage of the small municipalities is that they do not have a previously structured service network ${ }^{68,80}$. On the other hand, not only do larger municipalities require more organizational, political, and financial resources to reach good coverage, but they also provide care to individuals from other municipalities ${ }^{21,80,81}$. In fact, the 13 municipalities with less than 50\% Family Health Strategy coverage were large cities or metropolises, and 17 of the 21 municipalities with more than 70\% Family Health Strategy coverage were small.

In general, the municipalities with the lowest HDI-M had high Family Health Strategy coverage; low HDI-M is a primary requirement for the Ministry of Health to implement the strategy ${ }^{21}$.

Most studies were conducted in Brazilian Southeast municipalities mostly with low Family Health Strategy coverage. The Northeast region follows with the highest number of studies and highest mean Family Health Strategy coverage ${ }^{21}$. Since the approach regards food and nutrition and Family Health Strategy, the highest number of research groups in the Brazilian Southeast may explain the numerous articles. Search in the DGP confirmed the prevalence of the Southeast in the number of institutions with groups studying primary health care and the Family Health Strategy (Table 5). Nevertheless, numerous groups are also located in the Brazilian Northeast and South.

The information retrieved from the group and research line search confirm the scarcity of studies. Although a significant number of groups study food and nutrition in primary care, the number of groups that focus specifically on the Family Health Strategy is still small. However, focus on Family Health Strategy should be awakened and improved in view of the growing implementation of Núcleo de Apoio a Saúde da Família (NASF, Family Health Support Centers) and employment of dieticians in Brazilian municipalities after Ordinance $n^{\circ}$ 154/2008 was passed ${ }^{82}$.

Research Group Directory-based search has some limitations. Group selection depends on the detailed description of the researchers and respective keywords, and the latter are often used by many research lines. Therefore, it is possible that some groups appear as focusing on primary care when in fact they focus on Family Health Strategy. Another possibility is that some research groups left some fields blank when filling out the form, so they do not appear in any research line.

Time is also a limiting factor even in census-based searches because sometimes a group is inactivated and recertified by an institution, skipping a census. Also, it is not possible to distinguish between ongoing and completed studies in each research line unless the information is updated by the researchers.

Despite these weaknesses and need of improvement, this research tool is critical for disclosing the Brazilian food and nutrition research potential. 
Most studies are quantitative, indicating the prevalence of the positivistic approach over the understanding approach as a way of making the area of collective health scientifically legitimate ${ }^{83}$. However, there were many qualitative or mixed studies with solid methods, showing the academic effort (here, specifically the Brazilian) of making increasingly deep analyses in the health area $^{83}$.

Nevertheless, the food and nutrition actions in most studies were small and local, as shown by Tables 1, 2, and 3. This situation may impact professional practice. Although dieticians lead many studies, the number of nurses and physicians coordinating studies on food and nutrition was significant.

Among others, nurses have been protagonists in the establishment of the Family Health Strategy. But nurses accumulate many roles, including organizing the teamwork, and administrative and management activities ${ }^{75}$. Hence, the food and nutrition actions they create eventually appear ${ }^{75}$. Boog et al. ${ }^{84}$ mention that physicians and nurses find performing nutrition education activities challenging because they do not have the right background for this task and cannot deal with the subjective aspects associated with food intake.

Few studies mention dieticians in the Family Health Strategy teams or supporting them $35,42,47,77$, despite the fact that other studies have suggested their inclusion in such teams or their working in paralle ${ }^{56,78,85}$. Nurses' and physicians' limited education in food and nutrition, nurses' excessive workload in the Family Health Strategy, and the absence of a dietician may suggest the reason for food and nutrition actions appearing diluted in face of other actions more in line with the nurse's or physician's work.

The most common study topics and populations were diabetes mellitus/diabetics and high blood pressure/hypertensives. These individuals were often targeted by actions focusing on anthropometric assessment, salt/ sugar restriction recommendations, and prescriptive recommendations made mainly by physicians or nurses $38,42,46,56,69,75$. Although not provided routinely, education on healthier lifestyles and food habits was cited as the most effective primary control strategy, and sometimes the only control strategy $28,38,42,46,62$. In spite of the importance of chronic NCD in the Brazilian nosological landscape, the studies that covered NCD comprehensively and from the viewpoint of multiple sectors were scarce.

Breastfeeding and child care were the second most common topics. Mother and child care has been a governmental priority in public health since the 1940s, and the people's priority since the 1980s, when the people demanded the creation and establishment of health policies ${ }^{86}$. In 2006 child's health officially becomes a strategic area of the National Primary Health Care Policy ${ }^{14}$. Health professionals ${ }^{10}$ are responsible for healthpromoting and disease-preventing food and nutrition actions, including the promotion of breastfeeding, possibly increasing interest on this topic.

However, woman's health care is not yet comparable to the quality of child's health care. The number of prenatal visits, and the quality and extent of this kind of care are yet inadequate, contributing to the persistently high mother mortality rates ${ }^{86}$. Few studies focused on this life stage.

None of the studies sampled adolescents, probably because the public health policies for this population were only introduced in primary care recently. In 2007 the Ministry of Health, together with the Ministry of Education, made possible the institution of health-related actions through the Programa de Saúde na Escola (PSE, School Health Program $)^{87}$ and created a care guide and record in 200988. The Semana Anual de Mobilização Saúde na Escola (Annual Week for School Health Mobilization) began in 2012 with the topic 'preventing obesity', representing the launching of PSE actions ${ }^{89}$. This may encourage researchers to conduct Family Health Strategy food and nutrition studies on this population. 
Although they constitute the National Policy for Food and Nutrition guidelines ${ }^{15}$, the theme Sisvan was not approached by any study and the themes on welfare programs (Programas de Transferência de Renda) and the Human Right to Adequate Food (HRAF) were approached in only one study. The current context is the persistence of food insecurity ${ }^{5}$; health professionals' feeling of helplessness, little knowledge about their work, and the perceived inability to establish intersectoral cooperation when they come across this reality ${ }^{90}$; and the organizational challenge of coordinating the welfare programs and the programs that encourage access to social rights ${ }^{91}$. On the other hand, the Family Health Strategy has reach and allows professionals to access population needs ${ }^{15}$ that require further investigation.

Nearly all articles on management regarded assessment, so they were also interconnected with either control (service operation, degree of action implementation, and monitoring of the indicators of the target population) or organization (training and structure) ${ }^{10}$. Few studies approached planning and management aspects. These four elements must be balanced for the proper planning and coordination of nutritional care by the different care levels, units, and teams ${ }^{10}$.

The other intervention levels defined by the Matrix ${ }^{10}$ were mostly on nutritional diagnosis. This situation indicates how food and nutrition actions are restricted to immediate health problem resolution and not comprehensive enough to promote consistent health-promoting changes. Hence, nobody has yet used the principle of comprehensiveness care as a guide to health education actions that consider subjects emancipators and allow action coordination to flow intersectorally ${ }^{17}$.

Although focusing on primary care, a recent article ${ }^{92}$ mapped, systematized, and assessed the scientific production in nutrition, and its findings were similar to the present findings. The said article reviewed 117 studies; most used quantitative research methods $(n=106)$; were published in the last decade $(n=75)$; involved the Brazilian Southeast and South regions $(n=69)$; focused on nutritional diagnosis $(n=43)$; and sampled children $(n=45)$. Studies on HRAF and welfare programs were not found.

\section{CONCLUSION}

Studies on food and nutrition in the Family Health Strategy are occasional, and probably were conducted by a few research groups that explore the theme. Food and nutrition actions are limited and local. Greater interest lies in child healthrelated studies. Generally, most studies on the management of food and nutrition actions explored their control and organization, and studies on nutritional care focused on nutritional diagnosis.

In conclusion, authorities should foment studies that propose more widespread and consistent food and nutrition actions in the Family Health Strategy, especially family- and grouporiented actions that foster the principles of comprehensive health care.

\section{CONTRIBUTORS}

IAL VASCONCELOS sought the articles and research groups in the Research Group Directory, wrote the article, and organized the references. LMP SANTOS supervised the work, reviewed the published articles, guided the Research Group Directory search, and helped to write and review the manuscript.

\section{REFERENCES}

1. Instituto Brasileiro de Geografia e Estatística. Pesquisa de Orçamentos Familiares 2008-2009: antropometria e avaliação do estado nutricional de crianças, adolescentes e adultos no Brasil. Rio de Janeiro: IBGE; 2010 [acesso 2012 jun 27]. Disponível em: $<$ http://www.ibge.gov.br/home/estatistica/ populacao/condicaodevida/pof/2008_2009/ POFpublicacao.pdf>. 
2. Brasil. Ministério da Saúde. Pesquisa Nacional de Demografia e Saúde da Criança e da Mulher - PNDS 2006: dimensões do processo reprodutivo e da saúde da criança. Brasília: Ministério da Saúde; 2009 [acesso 2012 jun 12]. Disponível em: <http:// bvsms.saude.gov.br/bvs/publicacoes/pnds_ crianca_mulher.pdf>.

3. Batista Filho M, Rissin A. Transição nutricional: tendências regionais e temporais. Cad Saúde Pública. 2003; 19(Supl 1):S181:S91. doi: 10.1590/S0102-311X 2003000700019

4. Instituto Brasileiro de Geografia e Estatística. Pesquisa de Orçamentos Familiares 2008-2009: análise do consumo alimentar pessoal no Brasil. Rio de Janeiro: IBGE; 2011 [acesso 2012 jun 25]. Disponível em: <http://www.ibge.gov.br/home/estatistica/ populacao/condicaodevida/pof/2008_2009_ analise_consumo/pofanalise_2008_2009.pdf>.

5. Instituto Brasileiro de Geografia e Estatística. Pesquisa nacional por amostra de domicílios: segurança alimentar 2004/2009. Rio de Janeiro: IBGE; 2010 [acesso 2013 jan 31]. Disponível em: <http://www. ibge.gov.br/home/estatistica/populacao/seguranca _alimentar_2004_2009/pnadalimentar.pdf>.

6. Instituto Brasileiro de Geografia e Estatística. Pesquisa por amostra de domicílios: um panorama da saúde no Brasil: acesso e utilização de serviços, condições de saúde e fatores de risco e proteção à saúde 2008. Rio de Janeiro: IBGE; 2010 [acesso 2012 jun 27]. Disponível em: <http://portal. saude.gov.br/portal/arquivos/pdf/pnad_2008_ saude_final.pdf>.

7. Brasil. Ministério da Saúde. Vigitel Brasil 2011: Vigilância de Fatores de Risco e Proteção para Doenças Crônicas por Inquérito Telefônico. Brasília: Ministério da Saúde; 2012 [acesso 2013 out 28]. Disponível em: <http://www.dive.sc.gov.br/ conteudos/agravos/Dant/VIGITEL-2011.pdf>.

8. Sartorelli DS, Franco LJ. Tendências do diabetes Mellitus no Brasil: o papel da transição nutricional. Cad Saúde Pública. 2003; 19(Supl 1):S29-S36. doi: 10.1590/S0102-311X2003000700004

9. Brasil. Ministério da Saúde. Obesidade. Brasília: Ministério da Saúde; 2006 [acesso 2012 jun 12]. Cadernos de Atenção Básica, n 12. Disponível em: <http://189.28.128.100/nutricao/docs/geral/doc_ obesidade.pdf>.

10. Brasil. Ministério da Saúde. Secretaria de Atenção à Saúde. Matriz das ações de alimentação e nutrição na Atenção Básica. Brasília: Ministério da Saúde; 2009 [acesso 2012 abr 15]. Disponível em: <http:// bvsms.saude.gov.br/bvs/publicacoes/matriz_ alimentacao_nutricao.pdf>.

11. Ferreira VA, Magalhães R. Nutrição e promoção da saúde: perspectivas atuais. Cad Saúde Pública.
2007: 23(7):1674-81. doi: 10.1590/S0102-31 $1 \times 2007000700019$

12. Coutinho JG, Gentil PC, Toral N. A desnutrição e obesidade no Brasil: o enfrentamento com base na agenda única da nutrição. Cad Saúde Pública 2008; 24(Supl 2):S332-S40. doi: 10.1590/S0102-31 $1 \times 2008001400018$

13. Brasil. Ministério da Saúde. Política nacional de promoção da saúde. $3^{a}$ ed. Brasília: Ministério da Saúde; 2010 [acesso 2012 jul 13]. Disponível em: <http://bvsms.saude.gov.br/bvs/publicacoes/ politica_nacional_promocao_saude_3ed.pdf>.

14. Brasil. Ministério da Saúde. Política nacional de atenção básica. Brasília: Ministério da Saúde; 2010 [acesso 2012 jul 13]. Série Pactos pela Saúde 2006. Disponível em: http://bvsms.saude.gov.br/bvs/ publicacoes/ politica_nacional_atencao_basica_ 2006.pdf.

15. Brasil. Ministério da Saúde. Política nacional de alimentação e nutrição. Brasília: Ministério da Saúde; 2012 [acesso 2012 jul 13]. Série B. Textos Básicos de Saúde. Disponível em: <http://189.28.128.100/ nutricao/ docs/geral/pnan2011.pdf>

16. Escoda MSQ. Para a crítica da transição nutricional. Ciênc Saúde Colet. 2002; 7(2):219-26. doi: 10.15 90/S1413-81232002000200002

17. Machado MFAS, Monteiro EMLM, Queiroz DT, Vieira NFC, Barroso MGT. Integralidade, formação de saúde, educação em saúde e as propostas do SUS: uma revisão conceitual. Ciênc Saúde Colet. 2007; 12(2):335-42. doi: 10.1590/S1413-81232 007000200009

18. Castro AA. Revisão sistemática e meta-análise, 2001. In: Elaboração e apresentação de comunicação científica. São Paulo: Metodologia; 2011 [acesso 2012 out 28]. Disponível em: <www. metodologia.org $>$.

19. Instituto Brasileiro de Geografia e Estatística. Contagem da população 2007. Rio de Janeiro: IBGE; 2007 [acesso 2012 out 28]. Disponível em: <http:// www.ibge.gov.br/home/estatistica/populacao/ contagem2007/>.

20. Brasil. Ministério da Saúde. Departamento de Informática do SUS (DATASUS). Indicadores do Pacto de Atenção Básica 2007. Brasília: Ministério da Saúde; 2007 [acesso 2012 jun 12]. Disponível em: <http://tabnet.datasus.gov.br/cgi/deftohtm. exe?pacto/2006/cnv/pacbr.def>.

21. Brasil. Ministério da Saúde. Saúde da família no Brasil: uma análise de indicadores selecionados: 1998-2005/2006. Brasília: Ministério da Saúde; 2008 [acesso 2012 nov 7]. Disponível em: <http:// 189.28.128.100/dab/docs/ publicacoes/geral/ saude_familia_no_brasil_uma_analise_indicadores_ selecionados_1998_2006.pdf>. 
22. Programa das Nações Unidas para o Desenvolvimento. Atlas do desenvolvimento humano do Brasil. Brasília: PNUD; 2000 [acesso 2012 jun 25]. Disponível em: <http://www.pnud.org.br/atlas/>.

23. Conselho Nacional de Desenvolvimento Científico e Tecnológico. Plataforma Lattes: currículo Lattes. Brasília: CNPq [acesso 2012 jun 27]. Disponível em: $<$ http://lattes.cnpq.br/>.

24. Conselho Nacional de Desenvolvimento Científico e Tecnológico. Plataforma Lattes: diretório dos grupos de pesquisa. Brasília: CNPq [acesso 2012 jul 15]. Disponível em:<http://dgp.cnpq.br/>.

25. Borges ALV, Philippi ST. Opinião de mulheres de uma unidade de saúde da família sobre a quantidade de leite materno produzido. Rev Latino-Am Enfer. 2003; 11(3):287-92. doi: 10.1590/S0104-11 692003000300004

26. Ferreira MLM, Ferreira LOC, Silva AA, Batista Filho M. Efetividade da aplicação do sulfato ferroso em doses semanais no Programa Saúde da Família em Caruaru, Pernambuco, Brasil. Cad Saúde Pública. 2003; 19(2):375:81. doi: 10.1590/S0102-311X200 3000200004

27. Sousa FGM, Araújo TL. Fatores de risco para carência nutricional de ferro em crianças de seis a sessenta meses na perspectiva do modelo campo de saúde. Rev Texto Contexto Enferm. 2004; 13(3): 420-6. doi: 10.1590/\$0104-07072004000300012

28. Mano GMP, Pierin AMG. Avaliação de pacientes hipertensos acompanhados pelo Programa de Saúde da Família em um Centro de Saúde Escola. Acta Paul Enferm. 2005; 18(3):269-75. doi: 10.1 590/S0103-21002005000300007

29. Parada CMGL, Carvalhaes MABL, Winckler CC, Winckler LA, Winckler VC. Situação do aleitamento materno em população assistida pelo Programa Saúde da Família-PSF. Rev Latino-Am Enferm. 2005; 13(3):407-14. doi: 10.1590/S0104-1169200500 0300016

30. Barbosa DL, Arruda IKG, Diniz AS. Prevalência e caracterização da anemia em idosos do Programa Saúde da Família. Rev Bras Hematol Hemoterap. 2006; 28(4):288-92. doi: 10.1590/S1516-84842 006000400014

31. Maria-Mengel MRS, Linhares MBM. Risk factors for infant development problems. Rev Latino-Am Enferm. 2007; 15(Esp):837-42. doi: 10.1590/S0 104-11692007000700019

32. Melo ASO, Assunção PL, Gondim SSR, Carvalho DF, Amorim MMR, Benicio MHD, et al. Estado nutricional materno, ganho de peso gestacional e peso ao nascer. Rev Bra Epidemiol. 2007; 10(2): 249-57. doi: 10.1590/S1415-790X200700020 0012
33. Nascimento AL, Diniz AS, Arruda IKG. Deficiência de vitamina A em idosos do Programa Saúde da Família de Camaragibe, PE, Brasil. ALAN. 2007; 57(3):213-8.

34. Alves JGB, Galé CR, Souza E, Batty GD. Efeito do exercício físico sobre peso corporal em crianças com excesso de peso: ensaio clínico comunitário randomizado em uma favela no Brasil. Cad Saúde Pública. 2008; 24(Supl 2):S353-9. doi: 10.1590/S0102-31 1X2008001400020

35. Azeredo CM, Maia TM, Rosa TCA, Silva FF, Cecon PR, Cotta RMM. Percepção de mães e profissionais de saúde sobre o aleitamento materno: encontros e desencontros. Rev Paul Pediatr. 2008; 26(4): 336-44. doi: 10.1590/S0103-0582200800040 0005

36. Assunção TS, Ursine PGS. Estudo dos fatores associados à adesão ao tratamento não farmacológico em portadores de diabetes Mellitus assistidos pelo Programa Saúde da Família, Ventosa, Belo Horizonte. Ciênc Saúde Colet. 2008; 13(Supl 2): 2189-197. doi: 10.1590/S1413-812320080009 00024

37. Cotta RMM, Batista KCS, Reis RS, Souza GA, Dias G, Castro FAF, et al. Perfil sociossanitário e estilo de vida de hipertensos e/ou diabéticos, usuários do Programa de Saúde da Família no município de Teixeiras, MG. Ciênc Saúde Colet. 2009; 14(4): 1251-60. doi: 10.1590/S1413-812320090004 00031

38. Girotto E, Andrade SM, Cabrera MAS, Graças Ridão E. Prevalência de fatores de risco para doenças cardiovasculares em hipertensos cadastrados em Unidade de Saúde da Família. Acta Sci Health Sci. 2009; 31(1):77-82. doi: 10.4025/actascihealthsci. v31i1.4492

39. Azeredo CM, Cotta RMM, Sant'Anna LFR, Franceschini SCC, Ribeiro RCL, Lamounier JA, et al. Efetividade superior do esquema diário de suplementação de ferro em lactentes. Rev Saúde Pública. 2010; 44(2):230-9. doi: 10.1590/S0034-8 9102010000200002

40. Brecailo MK, Corso ACT, Almeida CCB, Schmitz BAS. Fatores associados ao aleitamento materno exclusivo em Guarapuava, Paraná. Rev Nutr. 2010; 23(4):553-63. doi: 10.1590/\$1415-52732010000 400006

41. Felisbino-Mendes MS, Campos MD, Lana FCF. Avaliação do estado nutricional de crianças menores de 10 anos no município de Ferros, Minas Gerais. Rev Esc Enferm USP. 2010; 44(2):257-65. doi: 10.1590/S0080-62342010000200003

42. Silva LMC, Palha PF, Barbosa GR, Protti ST, Ramos AS. Aposentados com diabetes tipo 2 na Saúde da Família em Ribeirão Preto, São Paulo - Brasil. Rev 
Esc Enferm USP. 2010; 44(2):462-8. doi: 10.1590/S 0080-62342010000200031

43. Cristovão MF, Sato APS, Fujimori E. Excesso de peso e obesidade abdominal em mulheres atendidas em Unidade da Estratégia Saúde da Família. Rev Esc Enferm USP. 2011; 45(Esp 2):1667-72. doi: 10.15 90/S0080-62342011000800005

44. Garcia MT, Granado FS, Cardoso MA. Alimentação complementar e estado nutricional de crianças menores de dois anos atendidas no Programa Saúde da Família em Acrelândia, Acre, Amazônia Ocidental Brasileira. Cad Saúde Pública. 2011; 27(2):305-16. doi: 10.1590/S0102-311X201100 0200012

45. Cunha RM, Souza COS, Silva JF, Silva MA. Nível de atividade física e índices antropométricos de hipertensos e/ ou diabéticos de uma cidade do Brasil. Rev Salud Pública. 2012 [acesso 2013 out 17]; 14(3):429-37. Disponible en: <http://www. revistas.unal.edu.co/index.php/revsaludpublica/ article/download/27762/4026>

46. Marinho NBP, Vasconcelos HCA, Alencar AMPG, Almeida PC, Damasceno MMC. Diabetes Mellitus: fatores associados entre usuários da estratégia saúde da família. Acta Paul Enferm. 2012; 25(4): 595-600. doi: 10.1590/S0103-2100201200500 0018

47. Ribeiro AG, Cotta RMM, Silva LS, Ribeiro SMR, Dias CMGC, Mitre SM, et al. Hipertensão arterial e orientação domiciliar: o papel estratégico da saúde da família. Rev Nutr. 2012; 25(2):271-82. doi: 10.15 90/S1415-52732012000200009

48. Soares LDA, Campos FACS, Araújo MGR, Falcão APST, Lima BRDA, Siqueira DF, et al. Análise do desempenho motor associado ao estado nutricional de idosos cadastrados no Programa Saúde da Família, no município de Vitória de Santo Antão-PE. Ciênc Saúde Colet. 2012; 17(5):1297-304. doi: 10.1590/S1413-81232012000500023

49. Ferreira-Marim MM, Fabbro ALD. Estado nutricional avaliado por medidas antropométricas em préescolares atendidos pelo Programa de Saúde da Família de Ribeirão Preto, SP. Medicina. 2012; 45(1):23-30. doi: 10.11606/issn.2176-7262.v4 $5 \mathrm{i} 1 \mathrm{p}$

50. Vianna MVA, Cáder SA, Gomes ALM, Guimarães AC, Seixas-da-Silva IA, Rêgo ARON, et al. Aerobic conditioning, Blood Pressure (BP) and Body Mass Index (BMI) of older participants of the Brazilian Family Health Program (FHP) after 16 weeks of guided physical activity. Arch Gerontol Geriatr. 2012; 54(1):210-13. doi: 10.1016/j.archger.2011.0 2.013

51. Cesar JA, Cavaleti MA, Holthausen RS, Lima LGS. Mudanças em indicadores de saúde infantil em um município com agentes comunitários: o caso de Itapirapuã Paulista, Vale do Ribeira, São Paulo, Brasil. Cad Saúde Pública. 2002; 18(6):1647-54. doi: 10.1590/S0102-311X2002000600019

52. Ciconi RCV, Venancio SI, Escuder MML. Avaliação dos conhecimentos de equipes do Programa Saúde da Família sobre o manejo do aleitamento materno em um município da região metropolitana de São Paulo. Rev Bras Saúde Materno Infant. 2004; 4(2): 193-202. doi: 10.1590/S1519-38292004000200 010

53. Dubeux LS, Frias PG, Vidal SA, Santos DM. Incentivo ao aleitamento materno: uma avaliação das equipes de saúde da família do município de Olinda, Pernambuco. Rev Bras Saúde Mater Infant. 2004; 4(4):399-404. doi: 10.1590/S1519-38292004000 400009

54. Faleiros JJ, Kalil G, Casarin DP, Laque Jr. PA, Santos IS. Avaliação do impacto de um programa de puericultura na promoção da amamentação exclusiva. Cad Saúde Pública. 2005; 21(2):482-9. doi: 10.15 90/S0102-311X2005000200014

55. Roncalli AG, Lima KC. Impacto do Programa Saúde da Família sobre os indicadores de saúde da criança em municípios de grande porte da Região Nordeste do Brasil. Ciênc Saúde Colet. 2006; 11(3):713-24. doi: 10.1590/S1413-81232006000300018

56. Araújo JC, Guimarães AC. Controle da hipertensão arterial em uma unidade de saúde da família. Rev Saúde Pública. 2007; 41(3):368-74. doi: 10.1590/S 0034-89102007000300007

57. Caldeira AP, Aguiar GN, Magalhães WAC, Fagundes GC. Conhecimentos e práticas de promoção do aleitamento materno em Equipes de Saúde da Família em Montes Claros, Minas Gerais, Brasil. Cad Saúde Pública. 2007; 23(8):1965-70. doi: 10.1590/ S0102-311X2007000800023

58. Lima CC, Cavalcante AAM, Cotta RMM, Martins PC. Avaliação da assistência materno-infantil prestada por uma equipe rural do Programa Saúde da Família. Esc Anna Nery Rev Enferm. 2007; 11(3): 452-8. doi: 10.1590/S1414-81452007000300009.

59. Caldeira AP, Fagundes GC, Aguiar GN. Intervenção educacional em equipes do Programa de Saúde da Família para promoção da amamentação. Rev Saúde Pública. 2008; 42(6):1027-33. doi: 10.1590/ S0034-89102008005000057

60. Cesar JA, Mendoza-Sassi RA, Ulmi EF, Dall'Agnol MM, Neumann NA. Diferentes estratégias de visita domiciliar e seus efeitos sobre a assistência prénatal no extremo Sul do Brasil. Cad Saúde Pública. 2008; 24(11):2614-22. doi: 10.1590/S0102-31 $1 \times 2008001100016$

61. Costa GD, Cotta RMM, Reis JR, Siqueira-Batista R, Gomes AP, Franceschini SCC. Avaliação do cuidado 
à saúde da gestante no contexto do Programa Saúde da Família. Ciênc Saúde Colet. 2009; 14(Supl 1):1347-57. doi: 10.1590/S1413-81232009000 800007

62. Lima SML, Portela MC, Koster I, Escosteguy CC, Ferreira VMB, Brito $C$, et al. Utilização das diretrizes clínicas e resultados na atenção básica à hipertensão arterial. Cad Saúde Pública. 2009; 25(9):2001-11. doi: 10.1590/S0102-311X2009000900014

63. Niquini RP, Bittencourt SA, Lacerda EMA, Saunders C, Leal MC. Avaliação da estrutura de sete unidades de saúde da família para a oferta de assistência nutricional no pré-natal no município do Rio de Janeiro, Brasil. Rev Bras Saúde Mater Infant. 2010; 10(Suppl 1):561-8. doi: 10.1590/\$1519-38292010 000500006

64. Niquini RP, Bittencourt SA, Lacerda EMA, Saunders C, Leal MC. Avaliação do processo da assistência nutricional no pré-natal em sete unidades de saúde da família do município do Rio de Janeiro. Ciênc Saúde Colet. 2012; 17(10):2805-16. doi: 10.1590/S1 413-81232012001000028

65. Cervato-Mancuso AM, Tonacio LV, Silva ER, Vieira VL. A atuação do nutricionista na Atenção Básica à Saúde em um grande centro urbano. Ciênc Saúde Colet. 2012; 17(12):3289-300. doi: 10.1590/S14 13-81232012001200014

66. Cesar JA, Sutil AT, Santos GB, Cunha CF, MendozaSassi RA. Assistência pré-natal nos serviços públicos e privados de saúde: estudo transversal de base populacional em Rio Grande, Rio Grande do Sul, Brasil. Cad Saúde Pública. 2012; 28(11):2016-114. doi: 10.1590/S0102-311X2012001100010

67. Felisberto E, Carvalho EF, Maggi RS, Samico I. Avaliação do processo de implantação da estratégia da atenção integrada às doenças prevalentes da infância no Programa Saúde da Família, no estado de Pernambuco, Brasil. Cad Saúde Pública. 2002; 18(6):1737-45. doi: 10.1590/S0102-311X20020 00600028

68. Fernandez PMF, Voci SM, Kamata LH, Najas MS, Souza ALM. Programa Saúde da Família e as ações em nutrição em um distrito de saúde do município de São Paulo. Ciênc Saúde Colet. 2005; 10(3):749-55. doi: 10.1590/S1413-81232005000300031

69. Alves VS, Nunes M. Educação em saúde na atenção médica ao paciente com hipertensão arterial no Programa Saúde na Família. Interface. 2006; 10(9): 131-47. doi: 10.1590/S1414-328320060001 00010

70. Bustamante $V$, Trad LAB. Cuidando da saúde de crianças pequenas no contexto familiar: um estudo etnográfico com famílias de camadas populares. Ciênc Saúde Colet. 2007; 12(5):1175-84. doi: 10.1590/S1413-81232007000500014
71. Slomp FM, Mello DF, Scochi CGS, Leite AM. Assistência ao recém-nascido em um Programa de Saúde da Família. Rev Esc Enferm USP. 2007; 41(3):441-6. doi: 10.1590/S0080-62342007000 300014

72. Frota MA, Mamede ALS, Vieira LJES, Albuquerque CM, Martins MC. Práticas Culturais sobre aleitamento materno entre famílias cadastradas em um Programa de Saúde da Família. Rev Esc Enferm USP. 2009; 43(4):895-901. doi: 10.1590/S0080-6234 2009000400022

73. Pontes CM, Alexandrino AC, Osório MM. O envolvimento paterno no processo da amamentação: propostas de incentivo. Rev Bras Saúde Materinfant. 2009; 9(4):399-408. doi: 10.1590/\$1519-3 8292009000400003

74. Silva ACMA, Villar MAM, Wuillaume SM, Cardoso MHCA. Perspectivas de médicos do Programa Saúde da Família acerca das linhas de cuidados propostas pela Agenda de Compromisso para a Saúde Integral da Criança e Redução da Mortalidade Infantil. Cad Saúde Pública. 2009; 25(2): 349-58. doi: 10.159 0/S0102-311X2009000200013

75. Araújo JL, Paz EPA, Moreira TMM. Hermenêutica e o cuidado de saúde na Hipertensão Arterial realizado por enfermeiros na Estratégia de Saúde da Família. Esc Anna Nery Rev Enferm. 2010; 14(3): 560-6. doi: 10.1590/S1414-81452010000300018.

76. Pinto MS, Bosi MLM. Muito mais do que pe/(n)/ sam: percepções e experiências a cerca da obesidade entre usuárias da rede pública de saúde de um município do Nordeste do Brasil. Physis. 2010; 20(2):443-57. doi: 10.1590/S0103-7331201000 0200006

77. Ramos Cl, Cuervo MRM. Programa Bolsa Família: a interface entre a atuação profissional e o direito humano a alimentação adequada. Ciênc Saúde Colet. 2012; 17(8):2159-68. doi: 10.1590/S1413-812 32012000800026

78. Camossa ACA, Terarolli Junior R, Machado MLT. O fazer teórico-prático do nutricionista na estratégia saúde da família: representações sociais dos profissionais das equipes. Rev Nutr. 2012; 25(1):89-106. doi: 10.1590/S1415-52732012000100009

79. Cavalcante MGS, Samico I, Frias PG, Vidal SA. Análise de implantação das áreas estratégicas da Atenção Básica nas equipes de Saúde da Família em município de uma Região Metropolitana do Nordeste Brasileiro. Rev Bras Saúde Mater Infant. 2006; 6 (4):437-45. doi: 10.1590/\$1519-3829200600040 0011

80. Sousa MF. O Programa Saúde da Família no Brasil: análise do acesso à Atenção Básica. Rev Bras Enferm. 2008; 61(2):153-8. doi: 10.1590/S003471672008000200002 
81. Conill EM. Ensaio histórico-conceitual sobre a Atenção Primária à Saúde: desafios para organização dos serviços básicos e da Estratégia de Saúde da Família em centros urbanos no Brasil. Cad Saúde Pública. 2008; 24(Supl 1):S7-S27. doi: 10.1590/S0 102-311X2008001300002

82. Brasil. Portaria n¹54, de 24 de janeiro de 2008. Cria os núcleos de Apoio à Saúde da Família. Diário Oficial da União. 20084 mar; Seção 1; p.38-42.

83. Minayo MCS. O desafio do conhecimento: pesquisa qualitativa em saúde. 9a ed. São Paulo: Hucitec; 2006.

84. Boog MCF. Atuação do nutricionista em saúde pública na promoção da alimentação saudável. Rev Ciênc Saúde. 2008 [acesso 2013 out 17]; 1(1):33-42. Disponível em: <http://revistaseletronicas. pucrs.br/ ojs/index.php/faenfi/article/viewFile/3860/2932>.

85. Araújo LAO, Bachion MM. Diagnóstico de enfermagem do padrão mover idosos de uma comunidade atendida pelo Programa de Saúde da Família. Rev Escola Enferm USP. 2005; 39 (1):53-61. doi: 10.1590/S0080-62342005000100007

86. Santos Neto ET, Alves KCG, Zorzal M, Lima RCD. Políticas de Saúde Materna no Brasil: os nexos com indicadores de saúde materno-infantil. Saúde Soc. 2008; 17(2):107-9. doi: 10.1590/S0104-12902008 000200011

87. Brasil. Decreto $n^{\circ} 6.286$, de 5 de dezembro de 2007. Institui o Programa Saúde na Escola e cria outras providências. Diário Oficial da União. 2007 $6 \mathrm{dez}$.
88. Brasil. Ministério da Saúde. Caderneta de saúde do adolescente. Brasília: Ministério da Saúde; 2009 [acesso 2012 dez 12]. Disponível em: <http://portal. saude.gov.br/portal/arquivos/pdf/cardeneta_ meninos.pdf>.

89. Brasil. Ministério da Saúde. Passo a passo adesão a Semana Saúde na Escola. Brasília: Ministério da Educação; 2013 [acesso 2013 out 17]. Disponível em: <http://bvsms.saude.gov.br/bvs/publicacoes/ passo_adesao_semana_saude_escola.pdf $>$.

90. Pasquim EM. Nutrição no Programa de Saúde da Família [Especialização em Saúde Coletiva]. Brasília: Universidade de Brasília, 2002. In: Prêmio de Incentivo em Ciência e Tecnologia para o SUS. Edição 2002. Brasília: BVS; 2002 [acesso 2014 fev 3]. Disponível em: <http://bvsms.saude.gov.br/bvs/ct/ premio/2002_premiados.php>.

91. Santos LMP, Pasquim EM, Santos SMC. Programa de transferência de renda no Brasil: um estudo multidimensional da implementação do Bolsa Escola, Bolsa Alimentação e Cartão Alimentação. Ciênc Saúde Colet. 2011; 16(3):1821-34. doi: 10.1590/ S1413-81232011000300018

92. Canella DS, Silva ACF, Jaime PC. Produção científica sobre nutrição no âmbito da Atenção Primária à Saúde no Brasil: uma revisão da literatura. Ciênc Saúde Colet. 2013 18(2):297-308. doi: 10.1590/S1 413-81232013000200002

Received on: 25/22013 Version final on: 21/10/2013 Approved on: 26/11/2013 